\title{
Breaking the Rayleigh Limit in Thick Samples with Multi-slice Ptychography
}

\author{
Yi Jiang ${ }^{1}$, Zhen Chen $^{2}$, Ismail El Baggari ${ }^{1}$, Lena F. Kourkoutis ${ }^{2,3}$, Veit Elser ${ }^{1}$ and David A. Muller ${ }^{2,3}$
}

1. Department of Physics, Cornell University, Ithaca, NY.

2. School of Applied and Engineering Physics, Cornell University, Ithaca, NY.

${ }^{3 .}$ Kavli Institute at Cornell for Nanoscale Science, Ithaca, NY.

The development of a new generation of high-speed pixelated detectors has led to renewed interest in ptychography for electron microscopy. In contrast to conventional imaging, which is limited by the numerical aperture of the lens, ptychography with a high-dynamic range detector [1] can recover phase information from the full diffraction pattern. The projected potential of the specimen can then be retrieved to a resolution limited by the largest scattering angle and achieve much higher resolution and contrast than traditional imaging modes [2]. Current algorithms are based upon the strong phase approximation (SPA), which breaks down in the presence of strong dynamic scattering - resulting in a resolution reduction and contrast reversals in reconstructions even at moderate sample thicknesses, as shown in Figure 1.

Although a multi-slice ptychography approach [3], which divides the specimen into multiple layers, has been developed, it is rarely stable when reconstructing crystalline structures. First, the algorithm can easily converge to incorrect solutions because there are many more unknown variables than in SPA ptychography, while the input data (diffraction patterns) remains unchanged. Secondly, existing algorithms require precise knowledge of the sample thickness, which can require extensive matching to a large set of simulated diffraction patterns from known structures - problematic for unknown structures.

Here we modify the multi-slice ePIE algorithm to improve its convergence and address the thickness determination problem. The algorithm sets the distance between each slice to an integer multiple of the size of the unit cell, which can be determined from the measured HOLZ lines in the input data. In a crystal the projected potentials for each slice should then be exactly the same, and we only need to reconstruct a single transmission function for all slices - reducing the degrees of freedoms in the reconstruction problem. Instead of using a fixed total thickness, the algorithm begins with a few layers and dynamically adds additional slices to the reconstruction. For full diffraction patterns, the error between measured data and reconstruction reaches a minimum at the physical thickness and increases if more slices are added.

As an experimental demonstration, we imaged niobium chloride $\left(\mathrm{Nb}_{3} \mathrm{Cl}_{8}\right)$ at $120 \mathrm{keV}$ beam energy and 24 mrad aperture semi-angle. From multi-slice simulations the sample is estimated to be $10.7 \mathrm{~nm}$ thick. Down the [001] direction, the two $\mathrm{Nb}$ atoms are $0.67 \AA$ apart (Figure 2a), which is smaller than the $0.854 \AA$ Rayleigh limit, and not resolved in the ADF image (Figure $2 b$ ). While the SPA ePIE reconstruction shows the light $\mathrm{Cl}$ atoms, it fails to resolve the $\mathrm{Nb}-\mathrm{Nb}$ spacing, as the resolution is reduced by multiple scattering. In the multi-slice reconstruction, the two $\mathrm{Nb}$ atoms are resolved at the expected thickness (Figure 3d) exceeding the diffraction-limited resolution of the microscope [4].

\section{References:}

[1] M. Tate et al, Microscopy and Microanalysis 22 (2016), p. 237.

[2] Y. Jiang et al, arXiv: 1801.04630 [cond.Mat].

[3] A. M. Maiden et al, Journal of the Optical Society of America A 8 (2012), p. 1606. 
[4] Work supported by DOE (DE-SC0005827) \& NSF (DMR-1719875, DMR-1429155, DMR-1539918). $\mathrm{Nb}_{3} \mathrm{Cl}_{8}$ sample provided by J. P. Sheckelton, C. Pasco, T. M. McQueen (Johns Hopkins University).

$0.4 \mathrm{~nm}$

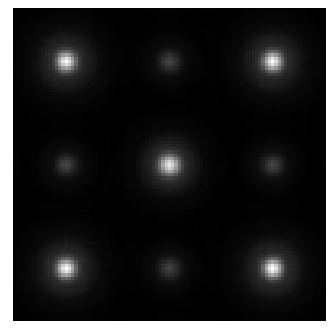

$2 \mathrm{~nm}$

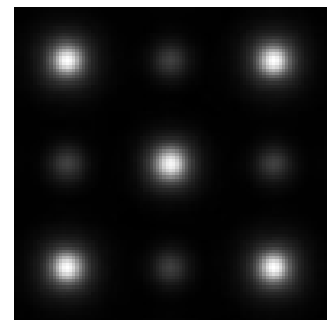

$8 \mathrm{~nm}$

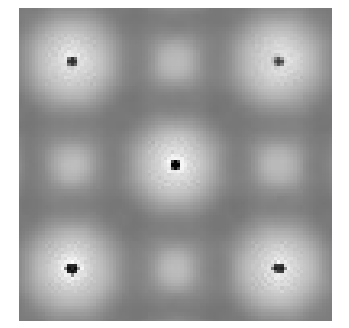

$16 \mathrm{~nm}$

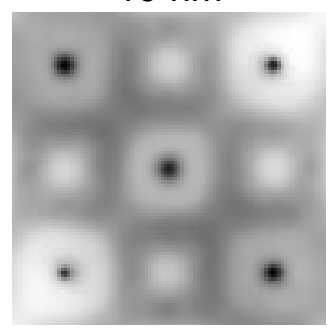

$24 \mathrm{~nm}$

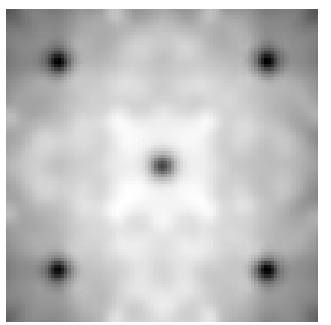

Figure 1. Ptychographic reconstructions from simulated $\mathrm{SrTiO}_{3} \mathrm{CBED}$ data for increasing thickness using the SPA-ePIE. As the sample thickness increases, standard ptychography initially shows degraded resolution, and eventually breaks down with distinct artifacts and contrast reversals.

(a)

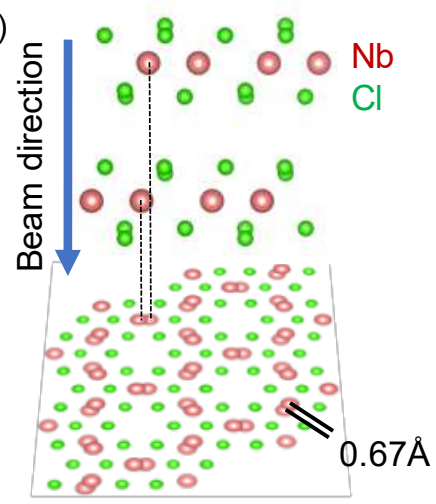

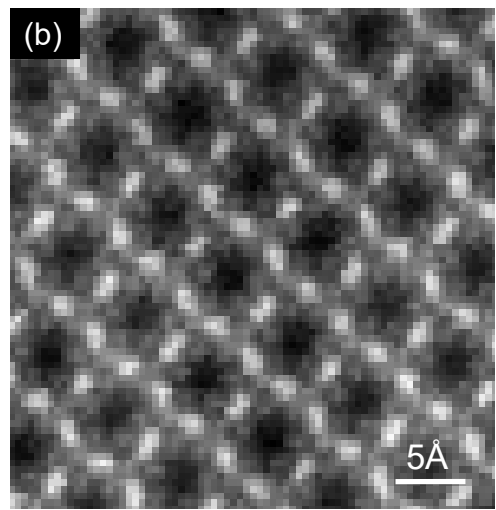

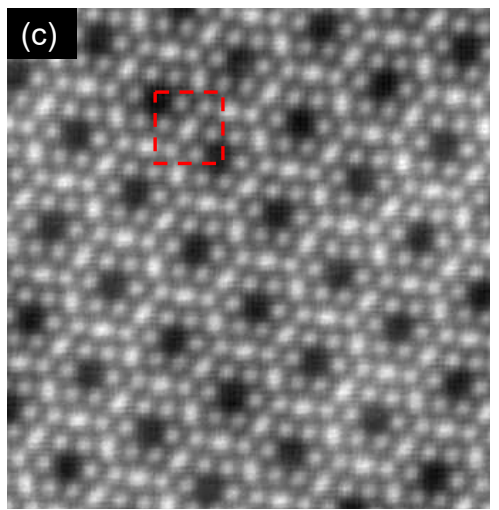

Figure 2. Ptychographic reconstruction of experimental data of $\mathrm{Nb}_{3} \mathrm{Cl}_{8}$. (a) The model structure along the [001] direction shows that the two $\mathrm{Nb}$ atoms are $0.67 \AA$ apart. (b) ADF image generated from the same 4D dataset. (c) Ptychographic reconstruction using the SPA-ePIE algorithm.
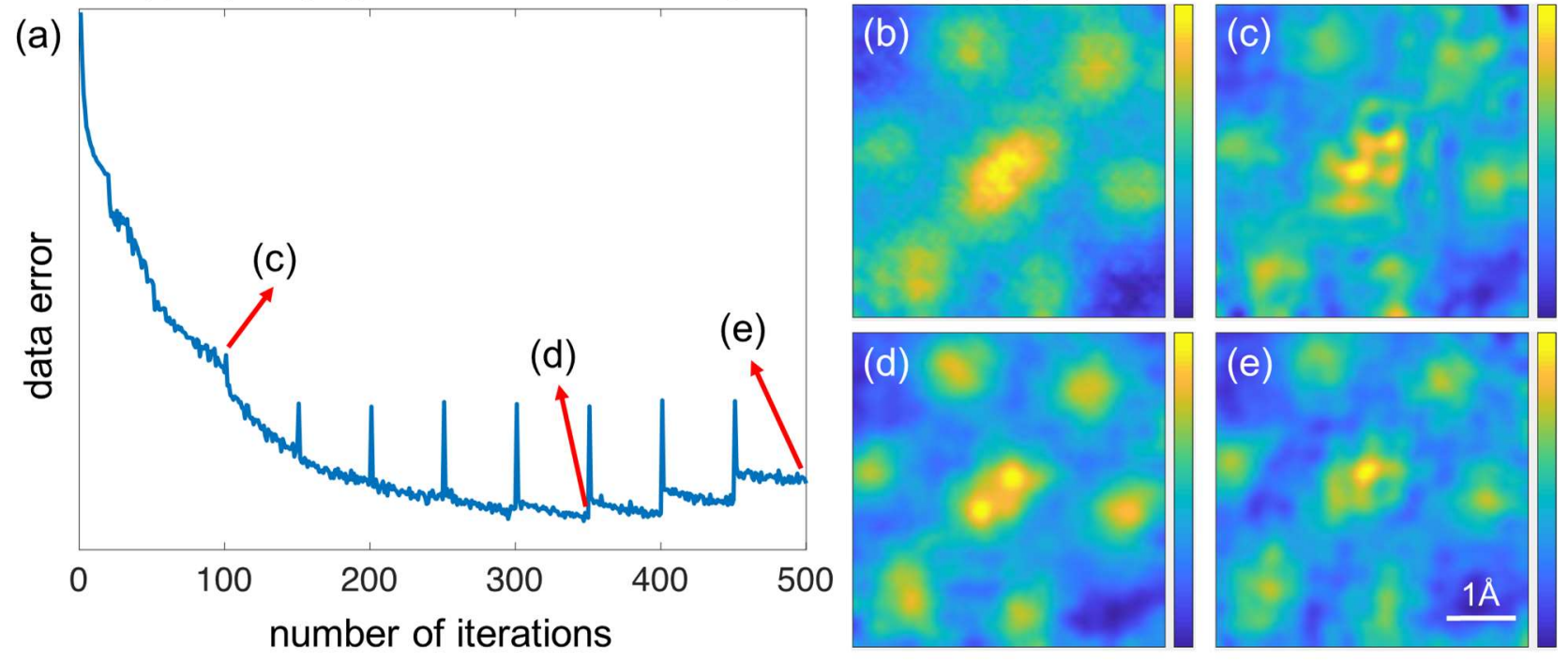

Figure 3. Multi-slice ptychographic reconstruction of experimental data of $\mathrm{Nb}_{3} \mathrm{Cl}_{8}$ (indicated by the red box in Figure 2c). (a) The error between measured data and reconstruction. A new slice is added to the reconstruction model for every 50 iterations. (b) Ptychographic reconstruction using the SPA-ePIE algorithm. (c-e) Multi-slice ptychographic reconstructions corresponding to a total thickness of $4.1 \mathrm{~nm}$, $10.9 \mathrm{~nm}$ and $15.0 \mathrm{~nm}$, respectively. The $\mathrm{Nb}$ atoms are resolvable when the correct thickness $(\mathrm{d})$ is reached. 